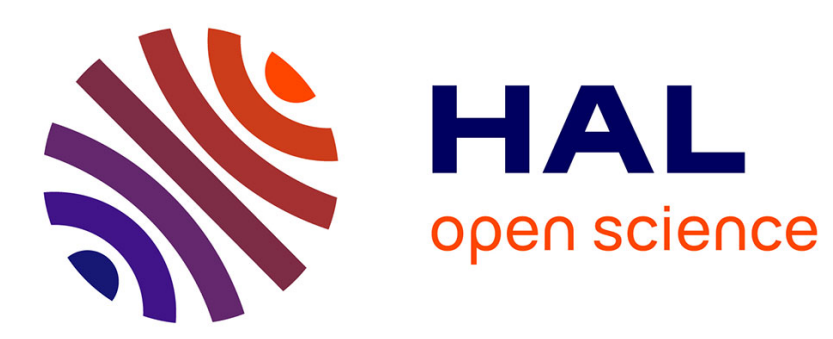

\title{
Identification of a nonlinear beam through a stochastic model based on a Duffing oscillator
}

\author{
Luis Gustavo Giacon Villani, Samuel da Silva, Americo Cunha Jr
}

\section{To cite this version:}

Luis Gustavo Giacon Villani, Samuel da Silva, Americo Cunha Jr. Identification of a nonlinear beam through a stochastic model based on a Duffing oscillator. 6th International Conference on Nonlinear Science and Complexity (NSC 2016), May 2016, São José dos Campos, Brazil. 10.20906/cps/nsc20160017 . hal-01471296

\author{
HAL Id: hal-01471296 \\ https://hal.science/hal-01471296
}

Submitted on 19 Feb 2017

HAL is a multi-disciplinary open access archive for the deposit and dissemination of scientific research documents, whether they are published or not. The documents may come from teaching and research institutions in France or abroad, or from public or private research centers.
L'archive ouverte pluridisciplinaire HAL, est destinée au dépôt et à la diffusion de documents scientifiques de niveau recherche, publiés ou non, émanant des établissements d'enseignement et de recherche français ou étrangers, des laboratoires publics ou privés. 


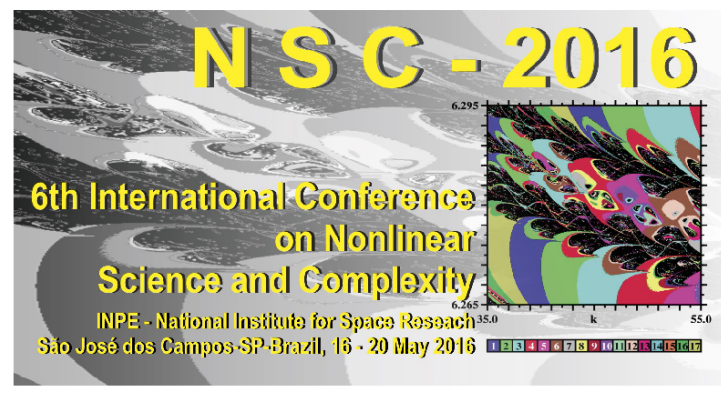

\author{
INPE - National Institute for Space Research \\ São José dos Campos - SP - Brazil - May 16-20, 2016
}

\title{
IDENTIFICATION OF A NONLINEAR BEAM THROUGH A STOCHASTIC MODEL BASED ON A DUFFING OSCILLATOR
}

\author{
Luis Gustavo Giacon Villani ${ }^{1}$, Samuel da Silva ${ }^{2}$, Americo Cunha $\mathrm{Jr}^{3}$ \\ ${ }^{1}$ UNESP - Univ Estadual Paulista, Faculdade de Engenharia de Ilha Solteira, Departamento de Engenharia Mecânica, Ilha Solteira, Brasil, \\ luisgustavovillani@gmail.com \\ ${ }^{2}$ UNESP - Univ Estadual Paulista, Faculdade de Engenharia de Ilha Solteira, Departamento de Engenharia Mecânica, Ilha Solteira, Brasil, \\ samuel@dem.feis.unesp.br \\ ${ }^{3}$ UERJ - Universidade do Estado do Rio de Janeiro, NUMERICO, Rio de Janeiro, Brasil, americo@ime.uerj.br
}

\begin{abstract}
This work proposes a model of a nonlinear beamshaker as a Duffing oscillator. The modal parameters of the linear equivalent systems and the nonlinear stiffness are estimated by stochastic methods considering the restoring force surface method. The parameters probability density functions (PDFs) are estimated of nonparametric way and a stochastic model is obtained with confidence limits to the responses. The model is validated with new experimental data through Monte-Carlo simulations. The results have been shown the capability to describe the nonlinear behavior of the beam considering uncertainties quantification.
\end{abstract}

keywords: Stochastic Models; Modeling, Numerical Simulation and Optimization; Nonlinear Dynamics and Complex Systems; Duffing Oscillator.

\section{INTRODUCTION}

The mathematical modeling of engineering structures is important to predict the dynamic systems behavior. Usually, the modal analysis process is used to describe the dynamic systems responses thorough the identification and validation of structural models based on the modal parameters (natural frequency, damping ratio and mode shapes)(e.g. [1]). Thus, models can be updated in order to know more deeply the behavior of the systems, and then optimize their operation.

Unfortunately, these approaches leads to good results for linear systems, because it is based on the superposition principle. However, it is known that many engineering structures have geometric and operating conditions, excitations and loads leading to highly nonlinear effects. Therefore, they should necessarily be considered in the analysis of the dynamic behavior of such structures (e.g. [2-4]]). The nonlinear systems have many individualities, so it is difficult to obtain a model that can describe all systems.

One of the first paper that proposed a nonlinear structures identification method was [5], which presents the method of restoring force surface. For nonlinear system with a single degree-of-freedom identification, the restoring force surface method is very effective. To better understand the structure behavior in nonlinear regime, it is useful to know the restoring force, i.e., a nonlinear function of system parameters.

On the other hand, the measurements have a lot of uncertainties related with ambient conditions, instruments and imperfections, among others. These variations are called data uncertainties and it is very important take into account these uncertainties in the model identification to ensure a better description of system behavior. So, the system uncertainties should be quantifying and limits of confidence should be established (e.g. [6-8]).

Thus, in this paper, the behavior of a free-clamped beam with nonlinear effects induced by the presence of a magnet near to the free end is approximated by a known literature benchmark, a Duffing oscillator (e.g. [9]). The modal parameters are identified by a stochastic way and their probability density functions (PDFs) are estimated by a nonparametric way for the linear and nonlinear motion regimes. With nonlinear motion regime, the cubic system stiffness is estimated of a stochastic way also, based on the restoring force surface method. After the identification of the PDFs, a stochastic model is constructed, with confidence limits to the system responses, and validated with new experimental data using Monte Carlo simulations. 


\section{EXPERIMENTAL SETUP}

The experimental setup is composed by an aluminum beam with dimensions of $300 \times 18 \times 3$ [mm] of length, as seen in Fig. 1. In the free end of the beam a steel mass was connected to cause an magnetic interaction between the beam and the magnet. A MODAL SHOP shaker (Model Number: $\mathrm{K} 2004 \mathrm{E} 01$ ) is attached $77 \mathrm{~mm}$ from the clamped and used to excite the structure considering different levels of voltage amplitude $0.05 \mathrm{~V}$ (low level), $0.10 \mathrm{~V}$ (medium level) and $0.15 \mathrm{~V}$ (high level). A laser (vibrometer) and a load cell were used to measure the velocity in the free end of the beam and the force excitation, respectively. All signals were measured considering a sampling rate of $1024 \mathrm{~Hz}$ and 4096 samples saved using a $m+p$ data acquisition system. It is important to note that the input signal considered was the voltage applied in the shaker as was made in [10]. This because the current applied in the shaker was kept constant but the force applied to the beam was not constant over a range of frequencies. Considering this, a relationship between the voltage applied in the shaker and the force applied to the beam was determined as $9 \mathrm{~N} / \mathrm{V}$.

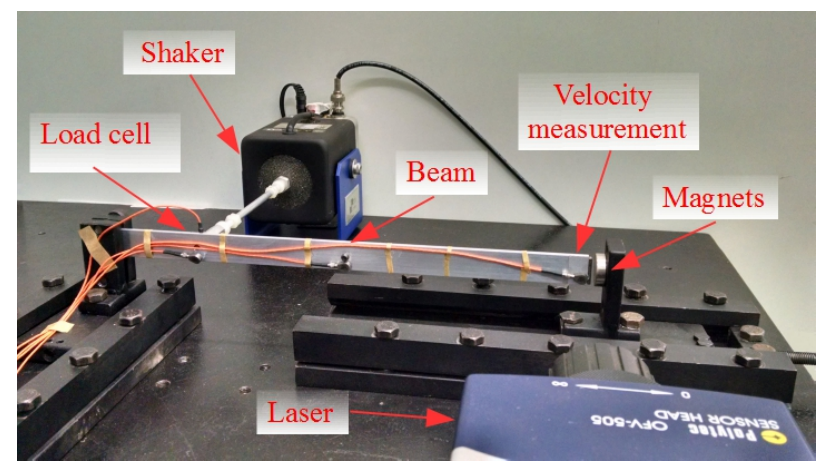

Figure 1 - Experimental setup.

The magnet interaction with the beam generates a nonlinear hardening behavior. This hardening effect is illustrated in the stepped sine test shows in Fig. 2. When the input signal is high, the beam presents a jump phenomenon that is represented by a sudden drop in the amplitude of the response. This results clearly shows the nonlinear behavior.

\section{MODEL CONSTRUCTION}

The studied system has nonlinear behavior for large displacements, therefore it can be approximated by single degree-of-freedom model represented by a Duffing oscillator (e.g. [9]). Considering that the parameters of motion equation have several uncertainties in their determination process, including measurements and data process uncertainties, the Duffing equation can be rewritten:

$$
\begin{array}{r}
\mathbb{m}(\theta) \ddot{\mathfrak{k}}(\theta, t)+\mathbb{C}(\theta) \dot{\mathfrak{k}}(\theta, t)+\mathbb{k}(\theta) \mathfrak{k}(\theta, t)+ \\
+\mathbb{F}_{n l}(\theta, \mathfrak{x}(\theta, t))=\mathbb{u}(\theta, t)
\end{array}
$$

where the symbol $\theta$ denotes an elementary event in the associated sample space, $m, \mathbb{C}$ and $\mathbb{k}$ are random variables that represents the equivalent mass $[\mathrm{kg}]$, damping coefficient

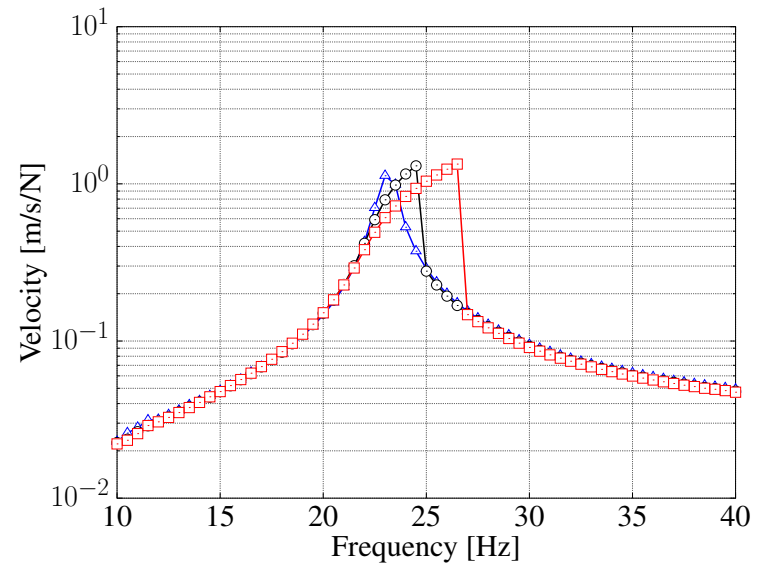

Figure 2 - Stepped sine test. Where $\triangle$ represents the low level of input signal $(0.05 \mathrm{~V})$, $\circ$ the medium level $(0.10 \mathrm{~V})$ and $\square$ the high level $(0.15 \mathrm{~V})$.

$[\mathrm{N} . \mathrm{s} / \mathrm{m}]$ and linear stiffness $[\mathrm{N} / \mathrm{m}]$ respectively, $u$ is the generalized force applied in the system $[\mathrm{N}]$ and $x$ is the displacement in the tip of the beam, the over-dots represent differentiation with respect to time. $\mathbb{F}_{n l}$ is the random restoring force $[\mathrm{N}]$, that in this case can be approximated by:

$$
\mathbb{F}_{n l}(\theta, \times(\theta, t)) \approx \mathbb{k}_{3}(\theta) \times(\theta, t)^{3}
$$

where $\mathbb{k}_{3}$ is the random nonlinear stiffness and $x$ is the displacement. If the cubic term is positive (negative), the nonlinear behavior of system is said to be hardening (softening).

\subsection{Parameters Identification Method}

To identify the parameters of system a lot of test were conducted. The beam has been excited by a chirp signal with range's frequency of 10 to $50 \mathrm{~Hz}$ and rate of $10 \mathrm{~Hz} / \mathrm{s}$, in two different levels of amplitude, low $(0.05 \mathrm{~V})$ and high $(0.15 \mathrm{~V})$, considering 620 repetitions in different days to construct the stochastic model.

The identification of linear parameters was done by an hybrid approach. First, we identified the damping ratio $(\S)$ and natural frequency $\left(\omega_{n}\right)$ of the equivalent linear system, considering low level of input $(0.05 \mathrm{~V})$, because in this condition the system has linear behavior. These parameters were identified through the experimental frequency response function (FRF) in the zone of first mode. The equivalent mass (m) was identified with an optimization process of the system response in the time domain and linear regime of motion. Finally, the damping coefficient $(\mathbb{C})$ and the linear stiffness $(\mathbb{K})$ were obtained by the expressions:

$$
\begin{array}{r}
\mathbb{C}(\theta)=2 \S(\theta) \omega_{n}(\theta) \mathfrak{m}(\theta) \\
\mathbb{k}(\theta)=\mathfrak{m}(\theta) \omega_{n}(\theta)^{2}
\end{array}
$$

With the linear parameters identified and applying high level of input to the beam (nonlinear regime of motion) we can determined the restoring force $\mathbb{F}_{n l}$ by the expression (e.g. [5]):

$$
\begin{aligned}
\mathbb{F}_{n l}(\theta, \mathfrak{x}(\theta, t)) & =\mathbb{u}(\theta, t)-\mathfrak{m}(\theta) \ddot{\ddot{x}}(\theta, t)+ \\
& -\mathbb{C}(\theta) \dot{\mathfrak{k}}(\theta, t)-\mathbb{k}(\theta) \mathfrak{x}(\theta, t)
\end{aligned}
$$

In this paper, the velocity and excitation were measured, while the beam acceleration and displacement were esti- 
mated by frequency domain derivation and integration. Once the restoring force identified, can determine the random nonlinear stiffness approximating the curves by a cubic polynomial order.

\section{IDENTIFICATION OF THE NONLINEAR BEAM}

This section presents the results that the authors have been obtained with the method described in section 3 applied in the experimental setup described in section 2 .

\subsection{Linear Vibration}

When the system has linear behavior, one can identify the linear model parameters, following the procedure described in section 3.1. For this, the structure was excited by a chirp signal from 10 to $50 \mathrm{~Hz}$ and rate of $10 \mathrm{~Hz} / \mathrm{s}$ with $0.05 \mathrm{~V}$ of voltage amplitude applied in the shaker. The test was repeated 620 times with sampling frequency of $1024 \mathrm{~Hz}$ and 4096 samples. Fig. 3 shows a nonparametric estimation of probability density function (PDFs) of linear parameters of the model $m, \mathbb{C}$ and $\mathbb{k}$. We can detect a multimodal behavior to each parameter.

\subsection{Nonlinear Vibration}

Now, when the system has nonlinear behavior we can identify the restoring force and the nonlinear stiffness. The structure was excited by the same chirp signal with $0.15 \mathrm{~V}$ of amplitude. The test was also repeated 620 times with sampling frequency of $1024 \mathrm{~Hz}$ and 4096 samples. Fig. 4 shows a nonparametric estimation probability density function (PDF) of the nonlinear stiffiness $\left(\mathbb{k}_{3}\right)$. We can see a large dispersion of the parameter that will influence the system response in nonlinear terms.

\subsection{Validation}

To validate the model new tests were done in the structure. The model was validated in time domain and frequency both in linear and nonlinear vibration regime. First, Fig. 5 shows the limits of confidence to the experimental and response of the stochastic model identified considering low and high level of amplitude voltage applied in the shaker. One can observe that the experimental response is inside the limits with 99\% of confidence what indicates that the model describe the system behavior in time domain for both linear as nonlinear behavior. The variation of response is consequence of the system variation and the uncertainties in the model parameters identification process. In frequency domain the model was validated with the stepped sine test. The test was made both with low as high input and the results are shown in Fig. 6 . The results shows that the model describes the system behavior both in linear as nonlinear regime of motion.

\section{FINAL REMARKS}

The results have showed that the studied system can be well approximated by a Duffing oscillator. The stochastic approach allows the model to represent the system behavior in different days and situations with statistical confidence.


(b) PDF of $\mathbb{C}$.

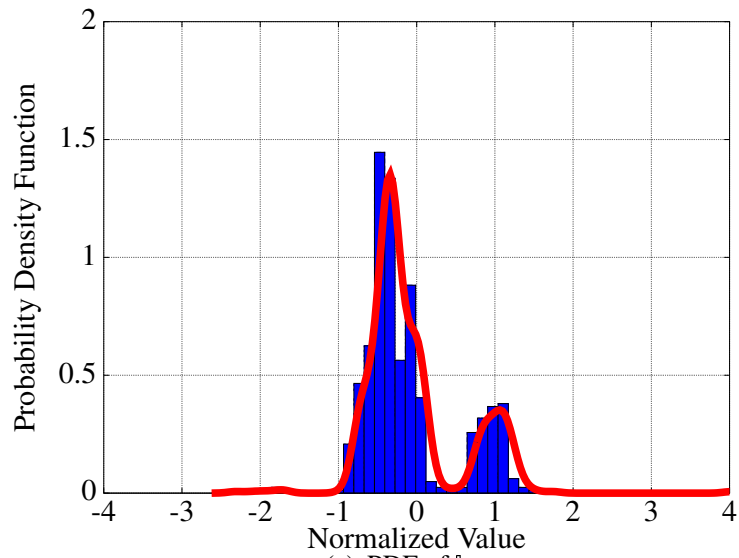

(c) PDF of $k$.

Figure 3 - Linear parameters normalized PDFs.

\section{ACKNOWLEDGMENTS}

The authors thank the financial support received from of São Paulo Research Foundation (FAPESP), grant number 2015/25676-2 and 2012/09135-3, and from Research Support Foundation of the State of Rio de Janeiro (FAPERJ), grant number E-26/010.002178/2015.

\section{References}

[1] P. Avitabile, "Experimental modal analysis," Sound and vibration, Vol. 35, No. 1, pp. 20-31, 2001.

[2] L. N. Virgin, "Introduction to experimental nonlinear dynamics: a case study in mechanical vibration," 


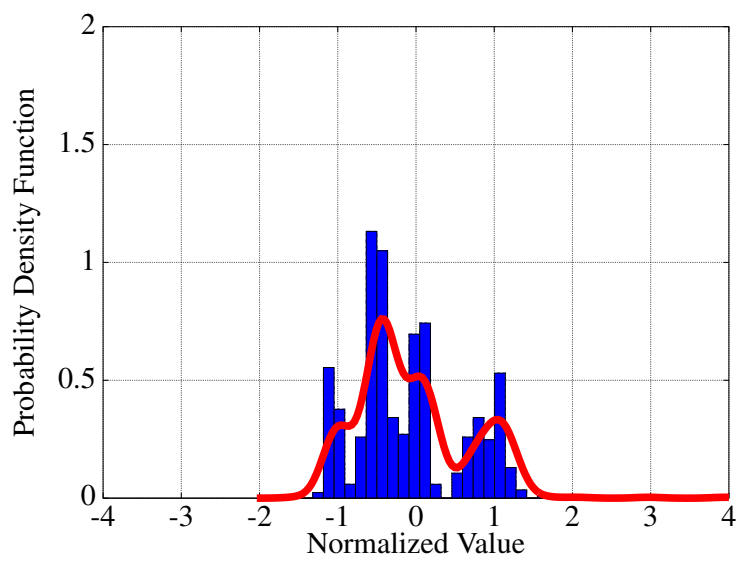

Figure 4 - Normalized PDF of $\mathbb{k}_{3}$.



(a) Low input.

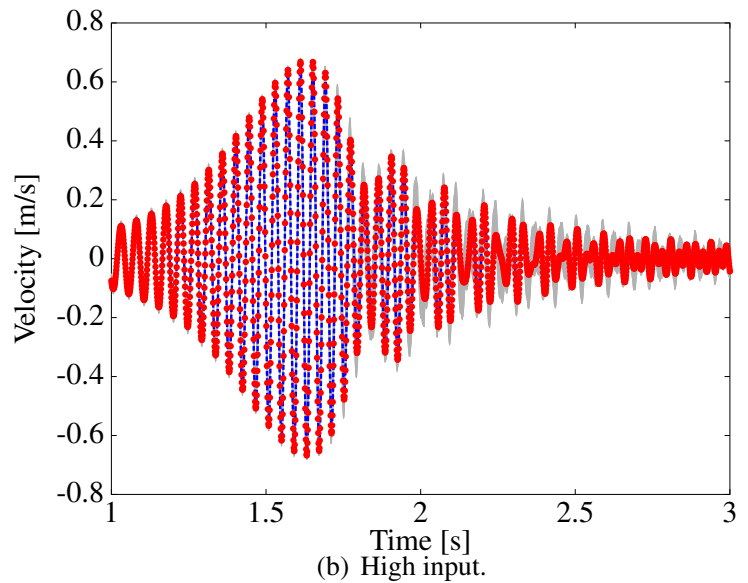

Figure 5 - System response with $99 \%$ of confidence. - represents the mean and $\circ$ the experimental data.

Cambridge University Press, 2000.

[3] K. Worden and G. R. Tomlinson, "Nonlinearity in structural dynamics: detection, identification and modelling," CRC Press, 2000.

[4] G. Kerschen, K. Worden, A. F. Vakakis and J. C. Golinval, "Past, present and future of nonlinear system identification in structural dynamics," Mechanical systems and signal processing, Vol. 20, No. 3, pp. 505592, April 2006.

[5] S. F. Masri, T. Caughey, "A nonparametric identifica-
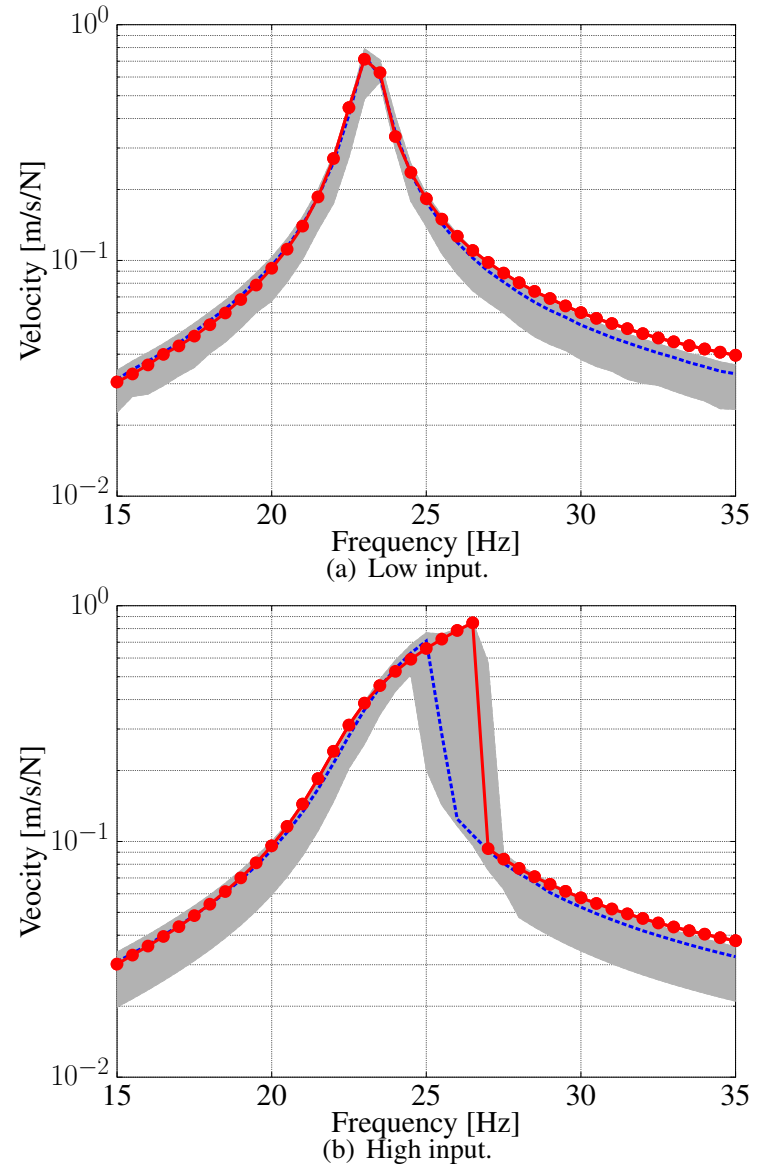

Figure 6-Stepped sine test with $99 \%$ of confidence. - represents the mean and $\circ$ the experimental data.

tion technique for nonlinear dynamic problems," Journal of Applied Mechanics, Vol. 46, No. 2, pp. 433-447, June 1979.

[6] C. Soize, "A comprehensive overview of a nonparametric probabilistic approach of model uncertainties for predictive models in structural dynamics," Journal of sound and vibration, Vol. 288, No. 3, pp. 623-652, December 2005.

[7] C. Soize, "Stochastic modeling of uncertainties in computational structural dynamicsâĂ Ťrecent theoretical advances," Journal of Sound and Vibration, Vol. 332, No. 10, pp. 2379-2395, May 2013.

[8] G. Iaccarino, "Quantification of uncertainty in flow simulations using probabilistic methods," Nato Research and Technology Organization Neuilly-SurSeine (France), September 2009.

[9] I. Kovacic, M. Brennan, "The Duffing equation: nonlinear oscillators and their behaviour," John Wiley \& Sons, 2011.

[10] B. Tang, et al. "Using nonlinear jumps to estimate cubic stiffness nonlinearity: An experimental study," Proceedings of the Institution of Mechanical Engineers, Part C: Journal of Mechanical Engineering Science, 2015. 